\title{
Fast Image Matching Algorithm Based on Pixel Gray Value
}

\author{
Jiawei Hu \\ School of North China Electric Power University, Baoding 071000, China \\ 798087749@qq.com
}

Keywords: Pixel Gray Value, Normalized Cross Correlation, Pyramid Hierarchical Search.

\begin{abstract}
Image matching technology is based on the template image in the search for the corresponding or similar target process in other images, which is a very important technology in the field of image information processing.In this paper, we use the matching algorithm based on pixel gray value, where the template matching method adopts normalized cross correlation(NCC) matching method, and adopt image pyramid hierarchical search strategy to improve the speed of image matching to achieve fast image matching.The algorithm is implemented by MATLAB software,and the results show that the method is feasible.
\end{abstract}

\section{Introduction}

Image matching technology is based on the template image in the search for the corresponding or similar target process in other images, which is a very important technology in the field of image information processing ${ }^{[1]}$.It can replace people to achieve the automation of image information collection and this technology has been widely used in the robot vision, aerospace, remote sensing image processing, medical image matching, aircraft cruise guidance and other fields .

At present, the practical image matching algorithm is divided into two categories: matching based on image geometric features and matching based on pixel gray value,and we choose the latter here which is a region-based approach,that is,considering the neighborhood properties of each matching point.It is usually matched by template, which has the advantages of high matching precision, and can improve the operation speed through other algorithms.Here, we adopt the image pyramid hierarchical search strategy to improve the image matching speed, the effect is remarkable!

The algorithm based on the grayscale correlation coefficient, is used to determine whether there is an image that is the same or similar to the template image in the target image by calculating the Grayscale correlation coefficient of the template image and the target image.The principle of the method is simple, easy to achieve, and in a good light conditions can be very satisfied with the matching results. We choose a relatively easy implementation of the template matching algorithm NCC here.

In summary ,the flow chart is shown in Fig.1:

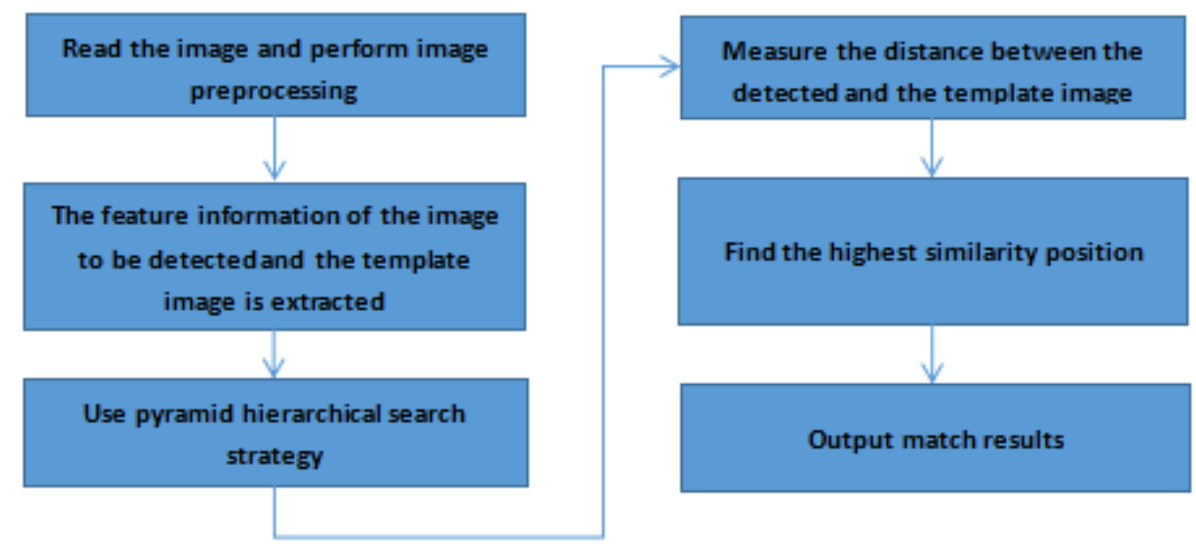

Figure.1 


\section{The algorithm is proposed}

\subsection{Normalized Cross Correlation(NCC) matching method ${ }^{[2][3]}$}

The simplest similarity measure is to calculate the squared sum of all the differences (SSD) between the template and the image.Set the template size is the pixels of $\mathrm{M} \times \mathrm{N}$.

Its function is as follows:

$$
S S D(i, j)=\sum_{m=1}^{M} \sum_{n=1}^{N}[T(m, n)-S(i+m, j+n)]^{2}
$$

Where $T(m, n)$ is the gray value of the template at the coordinate $(m, n)$ and $S(i+m, j+n)$ is the gray value of the searched image at the coordinate $(i+m, j+n)$.

Expand the Formula (1), we can get:

$$
\begin{aligned}
S S D(i, j)= & \sum_{m=1}^{M} \sum_{n=1}^{N}[T(m, n)]^{2} \\
& -2 \sum_{m=1}^{M} \sum_{n=1}^{N} T(m, n) S(i+m, s+n)+\sum_{m=1}^{M} \sum_{n=1}^{N}[S(i+m, j+n)]^{2}
\end{aligned}
$$

In the above equation, the first sum term of the equal sign on the right is the energy of the template,the second term is the cross-correlation between the template and the searched sub-image, and the third is the searched sub-image energy.Then,the above formula is normalized to get the template matching normalized correlation coefficient NCC:

$$
\operatorname{NCC}(i, j)=\frac{\sum_{m=1}^{M} \sum_{n=1}^{N} T(m, n) S(i+m, j+n)}{\sqrt{\sum_{m=1}^{M} \sum_{n=1}^{N}[T(m, n)]^{2}} \sqrt{\sum_{m=1}^{M} \sum_{n=1}^{N}[S(i+m, j+n)]^{2}}}
$$

Where the range of $\operatorname{NCC}(i, j)$ is $[0,1]$, and when the template and subgraph are exactly the same, the normalized correlation coefficient $\operatorname{NCC}(i, j)=1$.After completing all the searches in the searched image $S$, the maximum NCC value is found, and the corresponding sub-image is the matching target.

Some other functions that calculate similarity measures are:

$$
\operatorname{NCC}(i, j)=\frac{\sum_{m=1}^{M} \sum_{n=1}^{N}[T(m, n) S(i+m, j+n)]^{2}}{\sqrt{\sum_{m=1}^{M} \sum_{n=1}^{N}[T(m, n)]^{2}} \sqrt{\sum_{m=1}^{M} \sum_{n=1}^{N}[S(i+m, j+n)]^{2}}}
$$

The NCC value calculated according to formula(4) is close to zero when the template is similar to the sub-image.

$$
\operatorname{NCC}(i, j)=\frac{\sum_{m=1}^{M} \sum_{n=1}^{N}[T(m, n)-\bar{T} \llbracket S(i+m, j+n)-\bar{S}]}{\sqrt{\sum_{m=1}^{M} \sum_{n=1}^{N}[T(m, n)-\bar{T}]^{2} \sqrt{\sum_{m=1}^{M} \sum_{n=1}^{N}[S(i+m, j+n)-\bar{S}]^{\bar{T}}}}}
$$

Where $\bar{T} \backslash \bar{S}$ are the average gray values of the template and sub-image regions respectively.This formula is not sensitive to a small range of light changes in light. Therefore, we choose the above formula as a similar measure method in this paper.

\subsection{Search Strategy}

In order to find target matching the template from the image to be matched, a large range of searches is often required.Suppose that the size of the image to be matched is $N \times N$, and the matching template size is $M \times M(N>M)$. Then the average comparison of the two images by point comparison is $(N-M+1) \times(N-M+1) \times(M \times M) / 2$, and the amount of computation to be matched is huge.Therefore,we need to optimize the search strategy to improve the matching speed so as to reduce the amount of calculation. 
We adopt the image pyramid hierarchical search strategy ${ }^{[4]}$ in this paper.The basic idea is:the first thick after the fine match strategy.That is, firstly,the template image and the image to be matched are decomposed step by step to get a resolution from low to high,scale from small to large pyramid structure;Secondly,the rough matching is performed on the image layer with low resolution to obtain coarse position under low resolution.In the next level match, the result of the above level match is the guidance, so the matching is completed in the gradually smaller search area.As the resolution and matching accuracy are increased step by step, the matching of the accuracy requirements is finally achieved on the original image layer.

The flow chart of the matching strategy is shown in Fig.2:

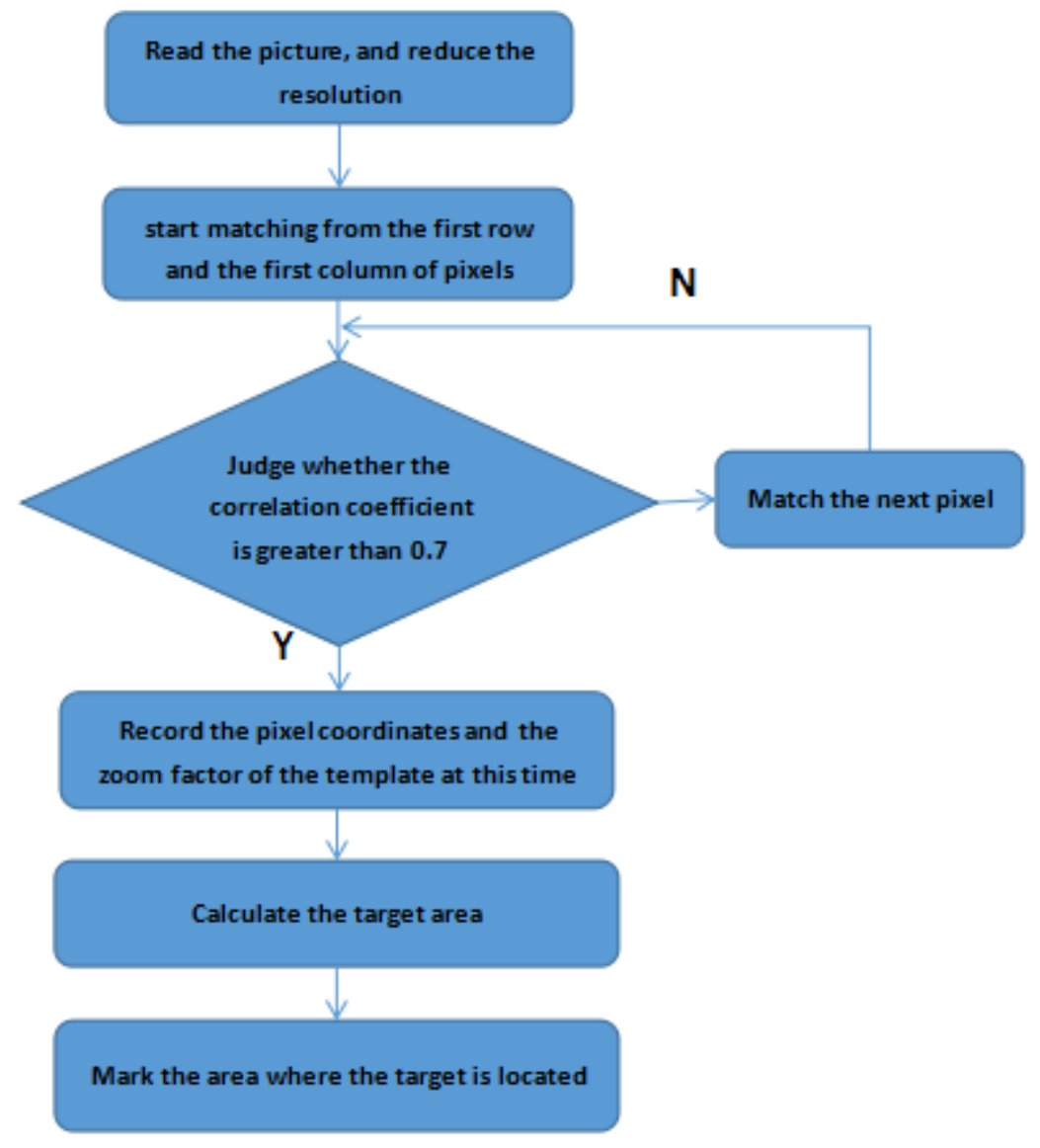

Figure.2

\section{Image Matching Example}

The pictures below shows the image matching result of the real shot image.Fig. 3 is the original image and matching results marked with a red box, Fig.4 is the corresponding template image. 


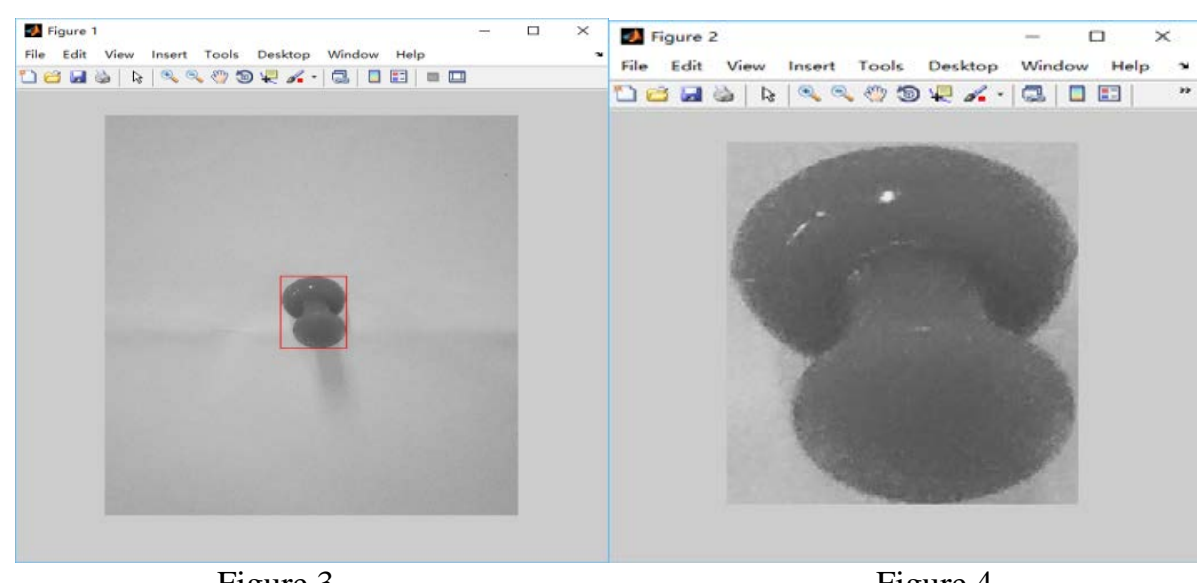

Experiments show that the error of the matching algorithm is controlled within the acceptable range.Besides, match all the pixels of the original image, the matching time $t_{1}=10.087317 \mathrm{~s}$, while the use of pyramid search strategy to prepare the program,the matching time $t_{2}=3.413456 \mathrm{~s}$.

\section{Conclusions}

In this paper, we use the matching algorithm based on pixel gray value, where the template matching method adopts normalized cross correlation matching method.Besides.we choose image pyramid hierarchical search strategy to improve the speed of image matching to achieve fast image matching and mark it out.Experimental comparison shows that the time spent on image matching can be shortened by nearly two thirds with the pyramid hierarchical search strategy which shows that this method has certain universality and engineering application value.

\section{References}

[1]Zhou Hui.Research and Implementation of fast image matching algorithm.May 2014

[2]Yang Tongyu,Peng Guohua.Fast image matching algorithm based on NCC[J].Modern Electronics Technique 2010, 22:107-109

[3]Sarvaiya J N, Patnaik S, Bombaywala S. Image registration by template matchingusing normalized cross-correlation[C]. Advances in Computing, Control, \&Telecommunication Technologies, 2009. ACT'09. International Conference on. IEEE,2009: 819-822

[4]Geng Guo zhang ,Yin Li min ,Lei Kai ,Wang Yan jie .An Improved Mean Pyramid Fast Search Algorithm.Chinese Journal Of Electron Devices,Vol.30 No.4 Aug.2007. 